Research Article

\title{
Biobjective Emergency Logistics Scheduling Model Based on Uncertain Traffic Conditions
}

\author{
Youqiang Sun, Yeqing Ren, and Xingjuan Cai $i$ \\ Complex System and Computational Intelligence Laboratory, Taiyuan University of Science and Technology, Taiyuan, \\ Shanxi 030024, China \\ Correspondence should be addressed to Xingjuan Cai; xingjuancai@163.com
}

Received 2 September 2019; Revised 8 November 2019; Accepted 19 December 2019; Published 25 January 2020

Academic Editor: Michele Guida

Copyright (C) 2020 Youqiang Sun et al. This is an open access article distributed under the Creative Commons Attribution License, which permits unrestricted use, distribution, and reproduction in any medium, provided the original work is properly cited.

\begin{abstract}
Emergency logistics scheduling appears more and more important in modern society because of frequent occurrence of unpredictable disasters. Most of the existing studies consider a certain emergency logistics scheduling model, and most of them are based on an ideal scenario. Considering the uncertain traffic condition and the real road condition, a biobjective emergency logistics scheduling model is proposed, which includes two objectives: transportation time and transportation cost. The uncertainty of the proposed model is reflected in two aspects: the occurrence time of emergencies and the traffic volume predicted by the cloud model. The numerical characteristics of traffic information are abstracted from the spatial-temporal trajectory data by the reverse cloud model, and the inference procedure of the one-dimension cloud model further predicts the uncertain traffic volume using the numerical characteristics. In addition, the crossover and mutation operators of multiobjective evolutionary algorithms are modified to solve the model. The experimental results show that the inference procedure of one-dimension cloud model can accurately predict the traffic volume at the departure time; and the proposed model is more reasonable than the existing scheduling models; at the same time, the improved NSGA-II can also provide superior schemes in different departure times and traffic conditions for decision makers.
\end{abstract}

\section{Introduction}

In recent years, natural disasters and man-made disasters happened frequently such as earthquake, typhoon, and fires, which seriously threaten the safety of people's life and property. In order to decrease the losses of the disasters, it is quite necessary to do the emergency rescue work well. As an important part of emergency rescue work, emergency logistics scheduling is a process that transports the emergency resource from the supply location to the disaster location. Emergency logistics scheduling usually has the characteristics of suddenness, unpredictability, uncertainty [1], urgency of time constraint, peak value, weak economy, and joint participation of the government and market. It is of great significance to construct a reasonable emergency logistics scheduling model based on real-world scenarios and obtain the optimal solution.
The objectives to be considered when establishing the emergency logistics scheduling model include minimizing the transportation time of resource, minimizing the transportation cost of resource, maximizing the satisfaction of people at the disaster location, and maximizing the satisfaction of drivers. Among them, minimizing transportation time and minimizing cost are the most concerned goals in scheduling, and many researchers established the emergency logistics scheduling model considering these two objectives [2-4]. The solving methods of emergency logistics scheduling model can be divided into two categories: one is mathematical analysis [5-9], such as the maximum flow and the minimum cost algorithm, which can effectively solve the emergency logistics scheduling problem and the results are relatively accurate. However, it is not suitable for solving the problems in the actual situation and there is a phenomenon of combinatorial explosion, but the intelligent optimization 
algorithm [10-14] can avoid such problems. Another is intelligent optimization algorithm [15-18], such as NSGAII. Since vehicle scheduling problem is proven to be NPcomplete problem by Savelsbergh [19], the research on emergency logistics scheduling problem has gradually shifted to seeking satisfactory solution for model and researchers tried to use intelligent optimization algorithm [20] to solve the emergency logistics scheduling model.

Different from the existing work, a biobjective emergency scheduling model based on uncertain traffic conditions was proposed to solve the actual scheduling problem, which takes minimizing transportation time and cost into consideration. The result shows the validity of this model, more reasonable than the existing models, and it also can provide more alternative emergency logistics scheduling schemes for decision makers. The contributions of this paper are as follows:

(1) The proposed model is established based on real road network and spatial-temporal trajectory data; the model is not a simple TSP $[21,22]$ problem; the route between supply locations and disaster locations is no longer ideal

(2) The uncertainty of the proposed model is reflected in two aspects: one is the fact that the one-dimension cloud model [23] transforms highly uncertain traffic information to roadway congestion index of road; on the other hand, the uncertainty of departure time leads to the uncertainty of traffic information

(3) The crossover and mutation operator of the multiobjective evolutionary algorithms [24-26] are improved in this paper, and then the improved algorithm is applied to work out the proposed model

The paper is arranged as follows. Section 2 gives the related works on emergency logistics scheduling and cloud model. A detailed description of the biobjective emergency logistics scheduling model based on uncertain traffic conditions is introduced in Section 3. Section 4 shows the experiments and results of the cloud model and scheduling model. The summary and next work are given in Section 5 .

\section{The Related Work}

Most of the emergency logistics scheduling models are solved by the mathematical analysis method. Chai et al. [27] considered the vehicle queuing phenomenon and used the rescue route travel time estimation to construct a traffic emergency resource scheduling, and the branch-bound algorithm is used to get the scheme after optimization. However, the author only considered the influence of travel time on scheduling when constructing the model, and the model is not reasonable enough. Ben-Tal et al. [6] proposed a robust optimization framework and applied the proposed framework to a contingency response and scheduling problem. But this framework does not consider the transportation cost; it still takes too long when solving the model. Li et al. [28] proposed a multisupply-locations and multidisaster-locations scheduling model, and a heuristic algorithm combining the network optimization and linear programming optimization is designed to solve the model. This model only considers the classification of multiresource but does not consider the time and cost in the scheduling process. Wang et al. [29] proposed a hybrid model based on the time-dependent network and robust discrete optimization theory to solve the emergency logistic network. Wang considers a single objective TSP problem, which is not applicable to real scheduling scenarios. Wang et al. [30] proposed a mathematical model based on the actual road, reliability analysis of the locations, edges, and topological structures; the model considers only ideal scenarios, but they did not take into account road congestion. The above models are all based on ideal scenarios, and they do not fully consider the problems in the real scheduling process, and these models are difficult to apply to real scenarios.

Traditional mathematical analysis methods have limited ability to solve with complex scheduling problems. However, intelligent optimization algorithms [31-34] have better ability in solving complex problems, especially in NP problems [35-38]. There are some intelligent optimization algorithms [39, 40] to solve the emergency logistics scheduling model. Ren et al. [41] proposed a multiperiod dynamic dispatching method considering the urgency and the connectivity reliability and adopted a hybrid genetic algorithm to solve the single-objective model. The uncertainty of rescue time and traffic conditions is not considered in this model; however, these factors are greatly affecting the efficiency of rescue. Deng et al. [42] presented a multiobjective robust vehicle routing problem with time windows, uncertain demand, uncertain driving time, and routing failure risk and adopted the improved SPEA2 to address this problem. Zhou et al. [43] proposed a multiobjective optimization model, including minimizing the unsatisfied demand of affected locations and minimizing the risk of choosing the damaged road, and improved the MOEA/D for solving the model. Deng and Zhou did not consider the real road network, and it is difficult to simulate the real scheduling environment. Chang [44] built an emergency logistics distribution vehicle scheduling model based on the division time, and the genetic algorithm and ant colony algorithm were combined for solving the vehicle scheduling model and parameter selection. However, the above research did not take road information into account when constructing the model. Ding et al. [45] proposed an effective emergency logistics scheduling model using congestion coefficient to describe road network condition. Different form Ding's method, the uncertainty of traffic information and rescue departure time are taken into account in our proposed model.

The models mentioned above do not consider the change of traffic volume. In this paper, the influence of traffic volume on scheduling is taken into consideration, and the spatial-temporal trajectory data are used to predict the traffic volume at the departure time. Most traffic forecasting methods, such as nonlinear theory, linear statistical method, and traffic simulation are often fragile and are not easy to be understood by nonprofessional users. The loss of traffic 
information samples will affect the prediction effect of these methods. However, the cloud model is more robust and easier to understand as a traffic prediction method. Further more, the single sample does not affect the overall numerical characteristics of the model, so the cloud model can effectively solve the problem of traffic information sample missing. Cloud model is an uncertain transformation model that can realize the transformation from the qualitative concept to quantitative data by using the probability statistics and fuzzy set theory and is proposed by $\mathrm{Li}[46,47]$. Many researchers have done traffic prediction based on cloud model. Yu et al. [48] proposed a traffic volume forecast algorithm that used the one-dimension cloud model, and it can effectively surmount the fuzziness and the randomness in traffic volume. Zhao et al. [49] developed a simplified computing method of sigmoid kernel based on the cloud model for predicting a real-time traffic information. Liu and $\mathrm{Xu}$ [50] proposed a short-term traffic flow prediction based on the cloud model and took Guangzhou traffic flow as an example; the simulation results showed that the prediction method is effective and advanced. Therefore, we can adopt the one-dimension cloud model to predict the uncertain traffic information.

In this paper, we used the cloud model to deal with uncertain traffic conditions. At the same time, the impact of road congestions and real road network are considered in scheduling when building the emergency logistics scheduling model.

\section{The Proposed Model}

In this section, the proposed biobjective emergency logistics scheduling model based on uncertain traffic conditions is introduced in detail. At the same time, we also briefly introduce the multiobjective evolutionary algorithm and the cloud model.

3.1. The Architecture of Scheduling Model. A biobjective emergency logistics scheduling model based on uncertain traffic conditions is constructed considering the two objectives: transportation time and transportation cost. The architecture of the scheduling model is shown in Figure 1. We extract numerical characteristics from Beijing's spatialtemporal trajectory data and real road network and build the corresponding cloud model. Through the qualitative reasoning of cloud model, the corresponding roadway congestion index can be obtained. At the same time, the multiobjective evolutionary algorithm is improved to solve the scheduling model. The process of the whole system is shown in Algorithm 1.

3.2. Problem Definition and Assumption. After the occurrence of disasters, a variety of factors are considered to establish an emergency logistics scheduling model. Suppose that, in an emergency rescue, the collection of supply locations and disaster locations is $i$ and $j$, respectively, and the amount of relief materials required is different at the different disaster locations. In order to match the supply location and disaster location and get the corresponding transportation route to minimize the total transportation time and cost, the optimal scheduling plans can be obtained by the established scheduling model to this scenario.

When an emergency occurs, each disaster location reports its disaster situation to the dispatching center; the center further analyses the disaster situation and gives relief commands to the supply locations; then the supply locations support each affected location according to the commands. In addition, the supply locations have enough relief materials to support the affected locations. Therefore, the following assumptions in the proposed model are made:

(1) The relief material demands of each disaster location are known, and the storage quantity of resources in each supply location is also known

(2) The supply location has enough transport vehicles available; meanwhile, the average speed of all transport vehicles from the supply point to the disaster point is the constant

(3) Transit stations are not considered in the scheduling process, and the relief materials are delivered from the supply location to the disaster location directly

(4) In the scheduling, roads are continuously available and traffic congestion remains unchanged

(5) In each rescue period, there is only one service from each emergency supply location to each disaster location

This paper takes Beijing as the background to study the problem of emergency logistics scheduling and abstract road network information into the corresponding vector map by using ArcGIS software and only major roads remained. There are 69 roads and 39 intersections in the vector map. All intersections and all roads are individually numbered to distinguish them. Then the real spatial-temporal trajectory data of Beijing are mapped to the vector map to get the traffic data of each road segment.

3.3. Biobjective Scheduling Model. There are two objectives in our model: transportation time and transportation cost. The definitions for each symbol in the model are shown in Table 1.

The first objective is the minimum total time of relief materials transportation, and the second objective is the minimum total cost of relief material transportation, defined as follows:

$$
\begin{aligned}
\min f_{1} & =\sum_{i \in S} \sum_{j \in D} \sum_{n \in N} \sum_{k \in K} \frac{d_{n}^{k} \cdot a_{n} \cdot b_{i j}}{\left(1-\lambda_{n}^{k}\right) v}, \\
\min f_{2} & =\sum_{i \in S} \sum_{j \in D} \sum_{n \in N} \sum_{k \in K} c \cdot d_{n}^{k} x_{i j} a_{n} \cdot b_{i j}, \\
a_{n} & = \begin{cases}1, & n=z(z \in N), \\
0, & n \neq z(z \in N),\end{cases}
\end{aligned}
$$




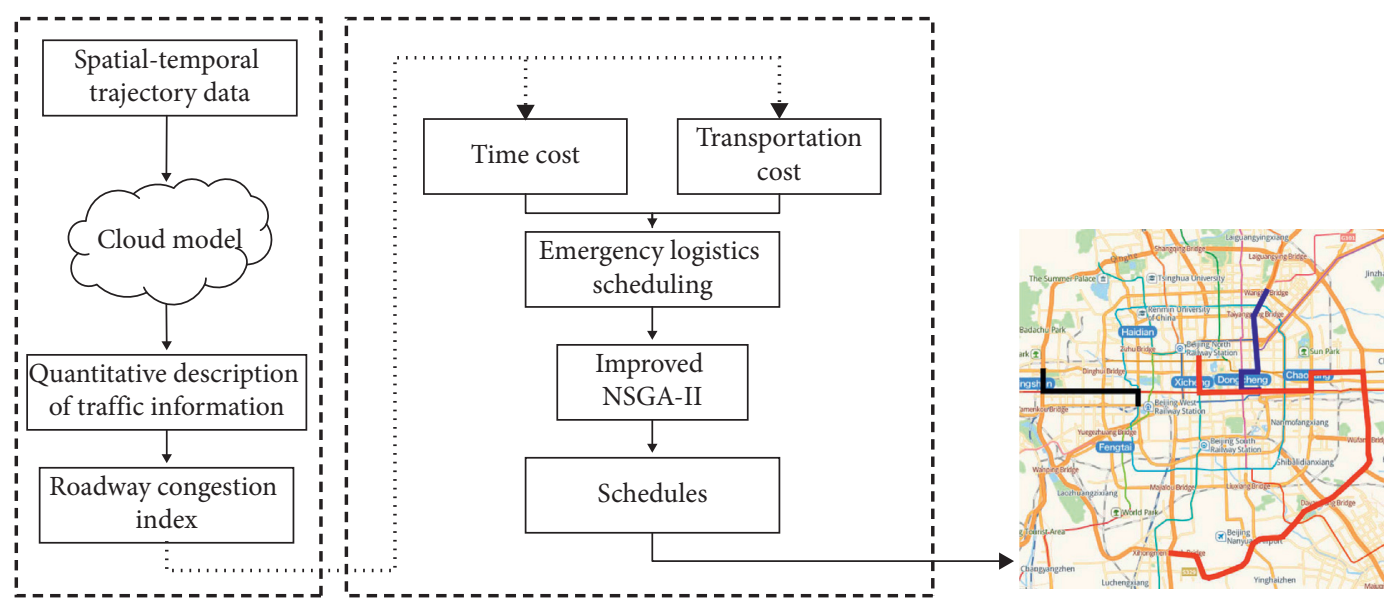

FIgURE 1: Biobjective emergency logistics scheduling model.
(1) Input the numerical characteristics and time
(2) Qualitative reasoning of the cloud model
(3) Output the traffic volume
(4) Convert the traffic volume to $\lambda$
(5) while $(i, j) \in$ supply point and disaster point
(6) Input the Initialized parameters and $\lambda$ to the scheduling model
(7) Initialize the population according to the adjacency matrix
(8) while $t<$ MaxGeneration
(9) Calculate fitness values and sort the $f_{1}, f_{2}$
(10) Tournament selection operation
(11) X-type crossover operation
(12) Point mutation operation
(13) Select the in individuals as the parents in next generation
(14) End
(15) End
(16) All fitness values are sorted by non-dominance
(17) All nondominant solutions are taken out as scheduling schemes

Algorithm 1: Biobjective emergency logistics scheduling model.

$$
\begin{aligned}
& b_{i j}= \begin{cases}1, & R_{i j}>0, \\
0, & \text { others, }\end{cases} \\
& x_{i j}=\left\lceil\frac{R_{i j}}{Q}\right\rceil .
\end{aligned}
$$

In equation (1), $\lambda_{n}^{k}$ is the roadway congestion index of the road segment $(k)$ in the plan $n$ from the supply location $i$ to the disaster location $j$. The higher the value of $\lambda_{n}^{k}$ is, the more congested the road is and the slower the driving speed is. The index of congestion varies at different departure times; therefore, logistics scheduling at different departure times is uncertain. $v$ is the average speed of a vehicle in the equation. $d_{n}^{k}$ is the physical length of the road segment $k$ in the route $n$. $a_{n}$ represents whether the route $n$ is the selected scheme. $b_{i j}$ represents the connectivity of the supply location $i$ to the disaster location $j$.

In equation (2), $c$ is the transportation cost for each vehicle and unit distance. $x_{i j}$ means the total number of vehicles from the supply location $i$ to the disaster location $j$. $d_{n}^{k}, a_{n}$, and $b_{i j}$ have the same meaning as equation (1).
There are some constraints in the above objectives:

(1) $a_{n}: z$ is the one chosen from $N$ scheduling routes; if the route $n$ is the selected route $z$, take 1; otherwise, take 0 , as equation (3)

(2) $b_{i j}$ : equation (4) is the connectivity between supply location $i$ and disaster location $j$ : can reach 1 and 0 otherwise

(3) $x_{i j}$ : equation (5) means the total number of vehicles from the supply location $i$ to the disaster location $j$, and $x_{i j}$ should satisfy the condition

In the conditions, $R_{i j}$ means the total amount of goods transported from the supply location $i$ to disaster location $j$; $Q$ is the maximum load of each vehicle; other parameters in the conditions have the same meaning as equations (1) and (2).

3.4. Roadway Congestion Index. In equation (1) of Section $3.3, \lambda$ is the roadway congestion index and the values can be obtained by this section. 
Table 1: Symbols and definitions.

\begin{tabular}{lc}
\hline Symbol & Definitions \\
\hline$S$ & A collection of supply locations $(i \in S)$ \\
$D$ & A collection of disaster locations $(j \in D)$ \\
$v$ & The speed of vehicles \\
$c$ & Transportation cost per vehicle with unit distance \\
$Q$ & Maximum load of vehicle \\
$N$ & A collection of the route plans from supply location $i$ \\
$n$ & to disaster location $j$ \\
$K$ & One route plan in $N(n \in N)$ \\
$k$ & A collection of all roads in a transportation plan from \\
$\lambda$ & supply location to disaster location \\
$d$ & A road segment of $K(k \in K)$ \\
$R_{i j}$ & The roadway congestion index of a road \\
& The physical length of a road segment \\
& The total amount of relief materials delivered from \\
& supply location $i$ to disaster location $j$
\end{tabular}

Most emergency logistics scheduling models consider the road in the ideal situation, for example, the Euclidean distance from the starting point to the ending point, and there is no traffic jam at the same time. But, in practical application, the roads in the ideal state are hard to exist. Congestion varies from road to road, while congestion on the same road is different at different times.

In the road network, the traffic volume usually has certain regularity and periodicity; the regularity reflected that the peak and low peak periods occur at roughly the same time every day, and the periodicity is reflected in the changing trend of traffic volume every week. Because most people's work is stable, congestion may occur at a fixed time on a fixed road section. A large number of vehicles will lead to peak traffic volume in the road network, which is mainly concentrated at the attendance time. Meanwhile, uncertain factors such as weather will also affect the traffic volume, so there is some randomness in the regularity and periodicity. Cloud model has randomness and fuzziness, which can represent the concept of traffic volume. The details of the cloud model are described in the following.

3.4.1. Cloud Model. Cloud model is an uncertain transformation model dealing with qualitative concepts and quantitative descriptions, which combines randomness and fuzziness. Cloud model can be summarized as follows:

$$
\mu_{C}(x): U_{C} \longrightarrow[0,1], \quad \forall x \in U_{C} \longrightarrow \mu_{C}(x) .
$$

Let $U$ be a quantitative domain set described by precise numbers and $C$ is a qualitative concept related to $U$, so $U_{C}$ means the quantitative domain set of the qualitative concept $C$. For any element $x$ in the domain $U_{C}$ of the qualitative concept $C$, there is a random number $\mu_{C}(x)$ with the stable tendency, and $\mu_{C}(x)$ is called as membership degree of element $x$ in the domain $U_{C}$. Then, the distribution of $x$ on domain $U$ is called cloud, and each $x$ is called a cloud drop. The range of values allowed for $\mu_{C}(x)$ is 0 to 1 . The cloud is a mapping from the domain $U$ to the unit interval $[0,1]$.

The numerical characteristics of the cloud model are represented by expected value $(E x)$, entropy $(E n)$, and hyperentropy $(\mathrm{He})$ in Figure 2. Expected value $E x$ is the expectation of cloud drops' spatial distribution in the whole domain, representing the point that can best represent the qualitative concept. Entropy En refers to the measure of uncertainty of qualitative concepts, which is determined by the randomness and fuzziness of concepts. Hyperentropy $H e$ is an uncertain measure of entropy, which is determined by the randomness and fuzziness of entropy; the higher the value, the greater the dispersion degree of entropy.

A series of samples $x_{i}$ of qualitative concept are obtained and $n$ is the number of samples, and their average value is obtained as the expectation Ex. The calculation formulas of expectation are as follows:

$$
E x=\frac{1}{n} \sum_{i=1}^{n} x_{i} .
$$

Meanwhile, the First-Order Absolute Central Moment of sample $x_{i}$ is calculated:

$$
\frac{1}{n} \sum_{i=1}^{n}\left|x_{i}-E x\right| .
$$

The Second-Order Absolute Central Moment is also calculated:

$$
S=\frac{1}{n-1} \sum_{i=1}^{n}\left(x_{i}-E x\right)^{2} .
$$

Therefore, the entropy En of the sample is calculated as follows:

$$
E n=\sqrt{\frac{\pi}{2}} \times \frac{1}{n} \sum_{i=1}^{n}\left|x_{i}-E x\right| .
$$

The calculation formula of the sample's hyper entropy $\mathrm{He}$ is as follows:

$$
H e=\sqrt{S-E n^{2}} \text {. }
$$

So, according to these steps, the numerical characteristics of qualitative concept are obtained: $(E x, E n, H e)$.

3.4.2. Traffic Volume Prediction. Since the characteristics of traffic volume can be completely represented by the cloud model, we construct the corresponding inference procedures. The traffic volume inference procedures based on cloud model consist of seven steps:

(1) Numerical characteristics are derived from the spatial-temporal trajectory data by the inverse Gaussian cloud model, and the numerical characteristics are assigned to various cloud models in the inference procedures.

(2) For each single rule, a one-dimensional random number $E n_{i}$ is generated with $E n_{A}$ as expectation and $H_{A}$ as variance, which conforms to one-dimensional normal distribution.

(3) According to the given time $t$, calculate the activation intensity $\mu_{i}$ of each former cloud generator according to (2); and $\mu_{i}=\exp \left[-\left((t-E x)^{2} / 2 E n_{i}^{2}\right)\right]$. 


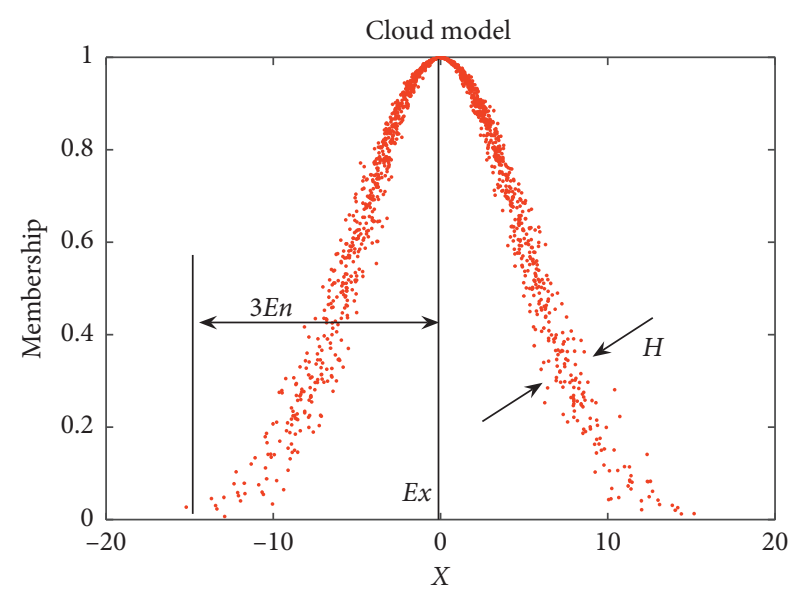

Figure 2: Three numerical characteristics in cloud model.

(4) The largest of $\mu_{i}$ should be selected; if there are more than two maximal values $\mu_{i}$, you can choose one of them. Then, activate the corresponding single rule, and generate a one-dimensional normal random number with $E n_{A}$ as expectation and $H_{A}$ as variance according to the given parameter $\mu_{i}$.

(5) According to the equation $E^{\prime} n_{B}=G\left(E n_{B}, H_{B}\right)$, generate a normal random number $E^{\prime} n_{B}$ with $E n_{B}$ as expected value and $H_{B}$ as standard deviation.

(6) Calculate $y$ by the equation $y=E x_{B} \pm \sqrt{-2 \ln (\mu)}$ $E^{\prime} n_{B}$; in the equation, the rising period determines the "+" and the "-" for falling period; then set $(y, \mu)$ as the cloud drop.

(7) Repeat several times until you get enough cloud drops, and finally output the average of all the drops.

The inference steps of the cloud model of traffic volume prediction are shown in Figure 3. The given time is input to the former cloud model PCG, and the membership corresponding to the time can be obtained; the corresponding memberships are input to the latter cloud model CG; then the predicted traffic volume is obtained.

The uncertainty is guaranteed in the inference procedure, because the membership $\mu$ generated by PCG randomly transfers the uncertainty in the domain $U_{\mathrm{PCG}}$ to the domain $U_{\mathrm{CG}}$, and $\mathrm{CG}$ outputs a random cloud drop under the control of $\mu$.

3.4.3. The Conversion of TD to RCI. Roadway congestion index (RCI) is determined by traffic density (TD), which refers to the density of vehicles in a road segment, that is, the number of vehicles per unit time and distance, also known as vehicle density.

Through the cloud model, the traffic volume of all streets at a certain moment can be obtained. According to the traffic volume, the RCI $(\lambda)$ can be obtained by the following equations:

$$
\begin{aligned}
\lambda_{i} & =\frac{\mathrm{TD}_{i}-\mathrm{TD}_{\min }}{\mathrm{TD}_{\max }-\mathrm{TD}_{\min }} \pm \varepsilon \\
\mathrm{TD}_{i} & =\frac{\mathrm{TV}_{i}}{L_{i}}
\end{aligned}
$$

From equation (12), $\lambda_{i}$ means the roadway congestion index for road segment $i$, TD is the traffic density, $\mathrm{TD}_{\max }$ is the highest density of all the road, $\mathrm{TD}_{\min }$ is the lowest density of all the road, and $\varepsilon$ is the minimum number, which is set to prevent $\lambda_{i}$ from reaching the boundary, "+" on the lower boundary, and "-" on the upper boundary. $\mathrm{TV}_{i}$ is the traffic volume, and $L_{i}$ is the physical length of the road segment $i$ in equation (13).

3.5. The Improved Multiobjective Algorithm. For solving the proposed uncertain emergency logistics scheduling model, this paper adopts the multiobjective evolutionary algorithm that is commonly used: NSGA-II. The model adopted in this paper is based on the real road and the collection of road segments through different routes is also different. So, the individual definition and operators of the standard multiobjective evolutionary algorithm NSGA-II are not applicable in our model. A new individual coding corresponding to crossover operator and mutation operator is designed.

3.5.1. Individual Coding. Firstly, we construct the corresponding adjacency matrix according to the connectivity of roads. When initializing an individual, the nonrepeating road set from the corresponding supply location to the disaster location is found by traversing the adjacency matrix. For example, we set the supply location (11) and the disaster location (15); a variety of the routes can be obtained; two of initialized individuals are shown in Table 2 ((a) and (b)).

3.5.2. Crossover Operator. Because of the continuity of elements in the individuals, in the crossover operation of the algorithms, the $\mathrm{x}$-type crossover is used. That is, randomly select an element in the intersection set of two different individuals, and use this element as the intersection point to conduct the crossover operation on two individuals.

For example, the same element 27 is selected from Table 2 (individuals a and $\mathrm{b}$ ) for $\mathrm{x}$-type crossover operation; the two individuals are divided into two segments by element 27 , and the two segments of different individuals are exchanged. The individuals after the crossover operation are shown in Table 3 ((a) and (b)).

3.5.3. Mutation Operator. Because the elements in each individual are related to the neighbouring elements, point variation is the only way to carry out mutation. A random mutation point is selected in an individual, and the individual is regenerated according to the adjacency matrix at the mutation point. For example, carry out mutation operation on individual (Table 2 (a)); assume that the selected mutation point is 27 , and regenerate new individuals from 


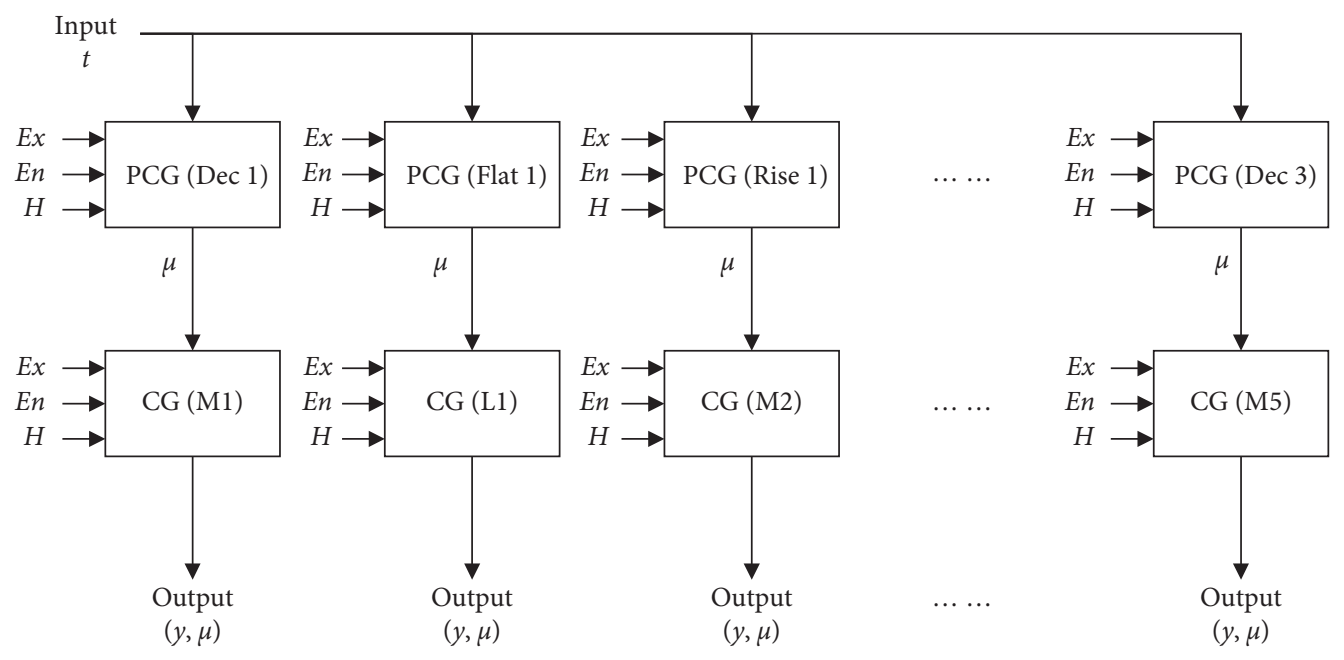

Figure 3: Qualitative reasoning of cloud model.

TABLE 2: Initialize individuals from the supply location (11) to the disaster location (15), such as path (a) and path (b).

\begin{tabular}{|c|c|c|c|c|c|c|c|c|c|c|c|c|c|c|c|}
\hline (a) & & & & & & & & & & & & & & & \\
\hline $\begin{array}{l}11 \\
\text { (b) }\end{array}$ & 10 & 19 & 27 & 18 & 17 & 16 & 7 & 6 & 5 & 4 & 13 & 22 & 23 & 14 & 15 \\
\hline 11 & 10 & 19 & 20 & 21 & 12 & 13 & 22 & 30 & 31 & 32 & 33 & 34 & 26 & 27 & 18 \\
\hline 9 & 8 & 17 & 16 & 25 & 24 & 15 & & & & & & & & & \\
\hline
\end{tabular}

TABle 3: Path (a) and path (b) after cross operation.

\begin{tabular}{|c|c|c|c|c|c|c|c|c|c|c|c|c|c|c|c|}
\hline (a) & & & & & & & & & & & & & & & \\
\hline (b) & & 11 & 10 & 19 & 27 & 18 & 9 & 8 & 17 & 16 & 25 & 24 & 15 & & \\
\hline 11 & 10 & 19 & 20 & 21 & 12 & 13 & 22 & 30 & 31 & 32 & 33 & 34 & 26 & 27 & 18 \\
\hline 17 & 16 & 7 & 6 & 5 & 4 & 13 & 22 & 23 & 14 & 15 & & & & & \\
\hline
\end{tabular}

the position of 27. The individuals before and after the mutation are shown in Table 4.

\section{Experiments and Results}

In this section, the experiment consists of two parts, respectively. First, $\lambda$ of 69 roads with the cloud model is solved. Then the time of scheduling departure is given; according to $\lambda$, the corresponding time scheduling is carried out.

4.1. Experiments of Cloud Model. The traffic data adopted in this paper are the Beijing taxi driving data. The total spatialtemporal trajectory data of Beijing reach 2.2 billion pieces. Due to the huge amount of data, we only kept the road network data of the major roads in Beijing and numbered the roads and intersections, with a total of 69 roads and 39 intersections. Among them, the grid method is adopted to filtrate the spatial-temporal trajectory data on the corresponding level of roads, and a one-dimensional cloud model is built with the filtered data. As the original spatial-temporal trajectory data are collected every minute, in order to more intuitively reflect the regular changes of traffic volume within a week, we take the average value of traffic information within 60 minutes as the data for an hour. The weekly traffic characteristics of one of the roads are shown in Figure 4.

As can be seen from the traffic volume in Figure 4, the traffic volume from Monday to Friday is similar, and the trends of Saturday and Sunday are roughly the same. There is a significant difference between weekday and weekend traffic volume, so we separate weekday traffic volume from weekend traffic volume to solve the change of traffic volume.

Table 5 generalizes the traffic volume and time attributes. Dec represents decline period, Flat represents low peak period, Rise represents rise period, and Peak represents peak period. $M$ is the traffic volume in the rising or falling period, $L$ is the traffic volume in the low peak period, and $\mathrm{H}$ is the traffic volume in the peak period, where the number after the letter indicates the number of times that this period occurs in one day. Table 6 shows the numerical characteristics of time cloud in nine different periods.

Because the cloud model requires numerical characteristics $(E x, E n$, and $H e)$ to represent concepts, the three characteristics from the data can be obtained by the inverse Gaussian cloud model. The numerical characteristics of time and traffic volume are shown in Tables 7-10.

In this paper, the traffic volume of the first week is used as the historical data, namely, Tables 7 and 8; and the second week is used as the current data, namely, Tables 9 and 10. In 
TABle 4: Path (a) after mutation operation.

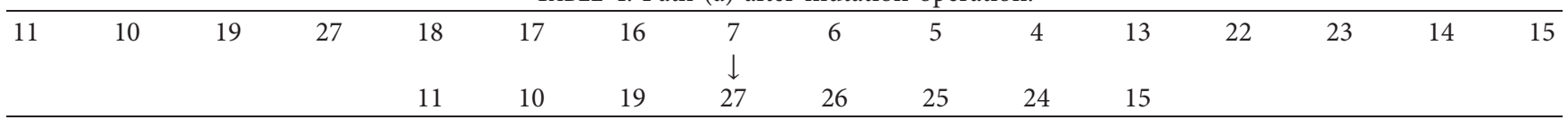

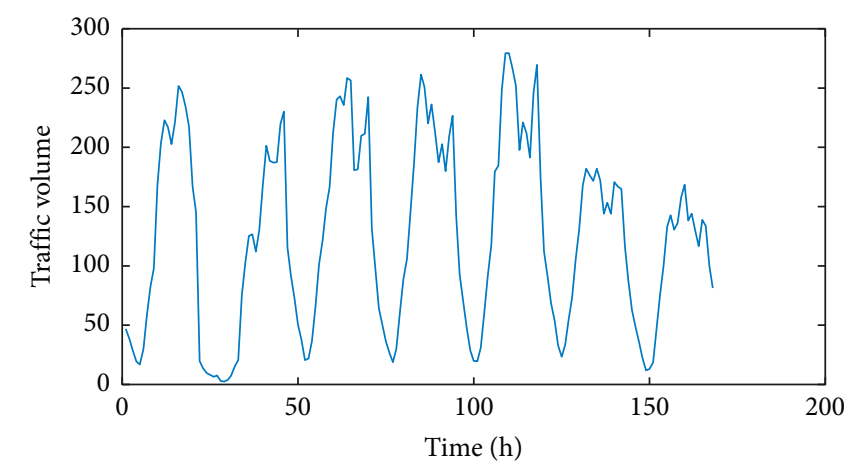

Figure 4: The traffic volume of the road for a week (Monday to Sunday).

TABLE 5: The generalized property.

\begin{tabular}{lccccccccc}
\hline Time & Dec 1 & Flat 1 & Rise 1 & Peak 1 & Dec 2 & Flat 2 & Rise 2 & Peak 2 & Dec 3 \\
\hline Traffic & M1 & L1 & M2 & H1 & M3 & L2 & M4 & H2 & M5 \\
\hline
\end{tabular}

TABLE 6: The numerical characteristics of time cloud model.

\begin{tabular}{lccccccccc}
\hline Para & Dec 1 & Flat 1 & Rise 1 & Peak 1 & Dec 2 & Flat 2 & Rise 2 & Peak 2 & Dec 3 \\
\hline Ex & 2.44 & 4.70 & 8.21 & 11.77 & 12.81 & 13.55 & 15.54 & 16.04 & 20.34 \\
En & 2.50 & 1.54 & 3.92 & 0.82 & 0.79 & 1.08 & 2.81 & 2.66 & 5.38 \\
He & 0.44 & 0.30 & 0.64 & 0.18 & 0.25 & 0.16 & 0.31 & 1.06 \\
\hline
\end{tabular}

TABLE 7: The numerical characteristics of the weekday history cloud.

\begin{tabular}{lccccccccc}
\hline Para & M1 & L1 & M2 & H1 & M3 & L2 & M4 & H2 & M5 \\
\hline Ex & 70.25 & 37.77 & 184.18 & 400.38 & 416.08 & 405.11 & 364.73 & 463.49 & 320.87 \\
En & 41.80 & 16.00 & 136.73 & 76.97 & 68.65 & 82.31 & 104.33 & 30.72 & 118.11 \\
He & 15.28 & 7.99 & 53.64 & 54.56 & 44.58 & 44.64 & 48.22 & 8.04 & 10.49 \\
\hline
\end{tabular}

TABLE 8: The numerical characteristics of the weekend history cloud.

\begin{tabular}{lccccccccc}
\hline Para & M1 & L1 & M2 & H1 & M3 & L2 & M4 & H2 & M5 \\
\hline Ex & 88.41 & 38.73 & 189.31 & 329.06 & 310.89 & 300.03 & 314.19 & 314.19 & 317.06 \\
En & 43.07 & 24.01 & 120.21 & 8.67 & 13.50 & 17.09 & 10.82 & 10.82 & 9.62 \\
He & 8.49 & 12.55 & 47.37 & 4.53 & 7.06 & 8.93 & 5.66 & 5.66 & 2.62 \\
\hline
\end{tabular}

TABLE 9: The numerical characteristics of the weekday current cloud.

\begin{tabular}{lccccccccc}
\hline Para & M1 & L1 & M2 & H1 & M3 & L2 & M4 & H2 & M5 \\
\hline Ex & 64.60 & 38.81 & 168.25 & 346.73 & 317.52 & 303.06 & 335.73 & 364.98 & 194.13 \\
En & 30.85 & 17.80 & 105.53 & 134.97 & 115.97 & 101.34 & 112.22 & 110.03 & 95.57 \\
He & 11.35 & 3.95 & 7.66 & 17.52 & 26.83 & 10.09 & 40.59 & 27.48 & 12.88 \\
\hline
\end{tabular}

the historical and current data, weekdays and weekends are separately processed, and then the mixed numerical characteristics of the two weeks are used as the hybrid cloud model.
With the stronger robustness of hybrid clouds, we fused the numerical characteristics of the two-week cloud model, as shown in Table 11. Table 12 shows the roadway congestion index $(\lambda)$ of 69 roads at 9 a.m. on Wednesday. The 
TABLE 10: The numerical characteristics of the weekend current cloud.

\begin{tabular}{lccccccccc}
\hline Para & M1 & L1 & M2 & H1 & M3 & L2 & M4 & H2 & M5 \\
\hline Ex & 50.26 & 5.34 & 56.41 & 182.99 & 178.22 & 172.05 & 219.75 & 256.63 & 208.77 \\
En & 26.28 & 6.69 & 63.96 & 8.17 & 17.30 & 25.04 & 44.03 & 65.54 & 34.40 \\
He & 7.50 & 3.50 & 19.82 & 4.27 & 9.04 & 13.09 & 9.11 & 34.26 & 14.96 \\
\hline
\end{tabular}

TABLE 11: The numerical characteristics of the hybrid cloud.

\begin{tabular}{lccccccc}
\hline & Time & \multicolumn{3}{c}{ Hybrid weekday cloud } & \multicolumn{3}{c}{ Hybrid weekend cloud } \\
Ex & En & He & Ex & En & He & Ex & En \\
\hline 2.44 & 2.50 & 0.44 & 67.85 & 72.65 & 13.61 & 73.95 & 69.35 \\
4.70 & 1.54 & 0.30 & 38.32 & 33.80 & 5.86 & 31.45 & 30.70 \\
8.21 & 3.92 & 0.64 & 177.24 & 242.26 & 33.61 & 143.16 & 184.17 \\
11.77 & 0.82 & 0.18 & 366.21 & 211.94 & 30.97 & 258.19 & 16.84 \\
12.81 & 0.79 & 0.25 & 354.17 & 184.62 & 33.43 & 236.37 & 30.80 \\
13.55 & 1.08 & 0.16 & 348.80 & 183.65 & 25.57 & 223.96 & 42.13 \\
15.54 & 2.81 & 0.31 & 349.70 & 216.55 & 44.27 & 238.38 & 54.85 \\
16.04 & 2.66 & 1.06 & 386.48 & 140.75 & 23.24 & 264.79 & 11.40 \\
20.34 & 5.38 & 1.01 & 264.18 & 213.68 & 11.56 & 232.44 & 76.36 \\
\hline
\end{tabular}

traffic volume of a consecutive week is predicted by the cloud model constructed based on the mixed numerical characteristics. Figure 5 shows the comparison between the hybrid prediction and the real traffic. From the result of prediction, the hybrid cloud model has a high accuracy in predicting the traffic volume for a consecutive week. Therefore, cloud model can be used for roadway congestion index prediction.

4.2. Experiments of Scheduling Model. The proposed model is a biobjective emergency logistics scheduling model considering multisupply locations and multidisaster locations. We selected three supply locations and three disaster locations in this experiment, and the selected location is shown in Figure 6(a). The selection of supply locations is from real large logistics center or large transport hub, and the disaster locations are selected from the shelters, and the distribution of supply locations and disaster locations should be even as far as possible to better display the experiment effect. Figure 6(b) is the vector map extracted from Figure 6(a) by using ArcGIS software.

When processing the spatial-temporal trajectory data, we found that the traffic volume trend of weekdays and weekends was quite different. In order to make the predictions more accurate, the data of weekdays and weekends were, respectively, processed, so the weekday and weekend clouds are obtained. In order to make the simulation experiments of proposed model be more representative, three different traffic conditions are selected. Three time-nodes are selected to represent different traffic conditions at 4 a.m. on Tuesday, at 9 a.m. on Wednesday, and at 9 a.m. on Saturday, respectively. 4 a.m. on Tuesday is a time point in a weekday when road conditions are more ideal; 9 a.m. on Wednesday is the more crowded time point in the weekday; 9 a.m. on Saturday is used to compare the differences between weekday and weekend. At the same time, the influence of different time points on the proposed model can also be reflected.
Three multiobjective algorithms are used in this paper, NSGA-II, MOEA/D, and SPEA2. The specific parameters of the algorithm are set in Table 13. The relief supplies of the disaster locations are shown in Table 14.

4.2.1. The Rescue Time at 4 a.m. on Tuesday. The morning of weekday (4 a.m. on Tuesday) is selected for the test, during which there were few vehicles on the road and most roads are in good conditions.

The second column of Table 15 shows the transportation times and costs. The first value of the bracket is the transportation time (in hours); and the second value refers to the cost (ten thousand yuan as unit). Table 16 is the average of the objectives, that is, the average consumption of time and cost in the transportation process, and it represents the general scheme and level. From Table 16, the results of NSGA-II and SPEA2 dominate the MOEA/D, so the solutions of NSGA-II and SPEA2 are better than the MOEA/D, while NSGA-II is $29 \%$ worse than SPEA2 in the first objective and NSGA-II is $54 \%$ better than SPEA2 in the second objective. In general, the schedules quality of NSGA-II is better than that of SPEA2.

In Table 17, NSGA-II can provide 4 plans for users to choose, while MOEA/D and SPEA2 provide 6 and 5 plans, respectively. However, there is no real Pareto front in the engineering problem; the metrics of $\mathrm{HV}$, spacing and PD, are not needed in the real Pareto front, so we select these metrics as the evaluation method. From the metrics that Table 17 provided, the HV measures the convergence of the algorithm, and the bigger is better; spacing measures the uniformity of the solution set, and the smaller is better; the diversity is calculated by PD, and the bigger is better; the number is the total number of schemes. SPEA2 has the best effect in the convergence of the algorithms but in the uniformity of the solution set, NSGA-II > MOEA/ D $>$ SPEA2. However, MOEA/D and NSGA-II have similar diversity of solution sets, both of which are better than SPEA2. 
TABLE 12: The RCI $(\lambda)$ of the 69 roads at 9 a.m. on Wednesday.

\begin{tabular}{|c|c|}
\hline No. & $\lambda$ \\
\hline 1 & 0.0576 \\
\hline 2 & 0.3492 \\
\hline 3 & 0.1918 \\
\hline 4 & 0.0262 \\
\hline 5 & 0.0188 \\
\hline 6 & 0.0477 \\
\hline 7 & 0.0789 \\
\hline 8 & 0.0341 \\
\hline 9 & 0.0984 \\
\hline 10 & 0.0720 \\
\hline 11 & 0.0917 \\
\hline 12 & 0.0341 \\
\hline 13 & 0.2689 \\
\hline 14 & 0.0712 \\
\hline 15 & 0.1730 \\
\hline 16 & 0.1203 \\
\hline 17 & 0.0260 \\
\hline 18 & 0.2462 \\
\hline 19 & 0.7915 \\
\hline 20 & 0.1625 \\
\hline 21 & 0.2495 \\
\hline 22 & 0.4519 \\
\hline 23 & 0.1176 \\
\hline 24 & 0.1944 \\
\hline 25 & 0.3744 \\
\hline 26 & 0.2614 \\
\hline 27 & 0.3498 \\
\hline 28 & 0.4188 \\
\hline 29 & 0.0538 \\
\hline 30 & 0.2356 \\
\hline 31 & 0.1067 \\
\hline 32 & 0.3013 \\
\hline 33 & 0.8236 \\
\hline 34 & 0.2673 \\
\hline 35 & 0.2257 \\
\hline 36 & 0.1467 \\
\hline 37 & 0.1232 \\
\hline 38 & 0.2952 \\
\hline 39 & 0.3140 \\
\hline 40 & 0.2853 \\
\hline 41 & 0.0100 \\
\hline 42 & 0.3552 \\
\hline 43 & 0.2871 \\
\hline 44 & 0.9900 \\
\hline 45 & 0.4675 \\
\hline 46 & 0.4153 \\
\hline 47 & 0.2438 \\
\hline 48 & 0.2266 \\
\hline 49 & 0.3383 \\
\hline 50 & 0.2931 \\
\hline 51 & 0.1913 \\
\hline 52 & 0.2329 \\
\hline 53 & 0.3937 \\
\hline 54 & 0.1322 \\
\hline 55 & 0.1941 \\
\hline 56 & 0.1775 \\
\hline 57 & 0.1617 \\
\hline 58 & 0.0867 \\
\hline 59 & 0.0767 \\
\hline 60 & 0.6277 \\
\hline 61 & 0.3818 \\
\hline
\end{tabular}

TABLE 12: Continued.

\begin{tabular}{lc}
\hline No. & $\lambda$ \\
\hline 62 & 0.2821 \\
63 & 0.0222 \\
64 & 0.3049 \\
65 & 0.6041 \\
66 & 0.0544 \\
67 & 0.0804 \\
68 & 0.2366 \\
69 & 0.0334 \\
\hline
\end{tabular}

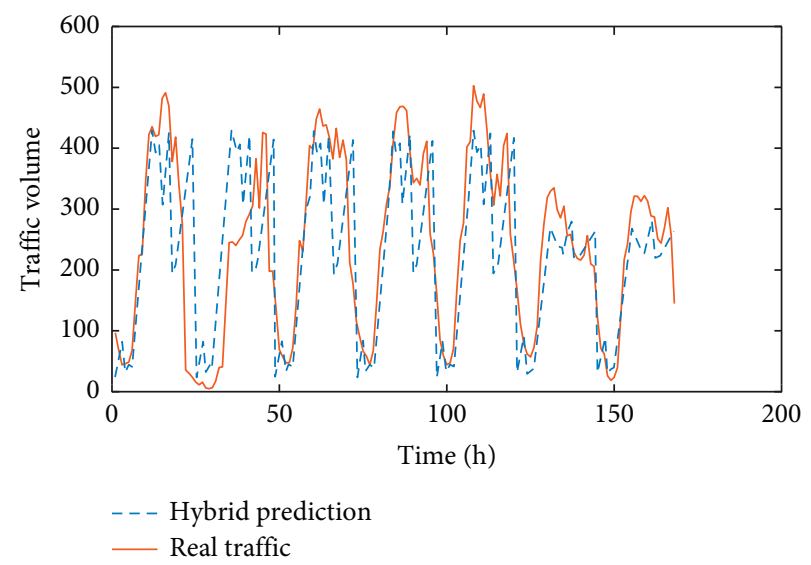

FIgURE 5: Forecasting traffic volume for a consecutive week.

In Figure 7, we can see that there are only a few points on the Pareto front, which is because there are many individuals clustered at a limited number of points. These several points dominate the other solutions that have been found; in other words, these points are better than any other solutions.

4.2.2. The Rescue Time at 9 a.m. on Wednesday. The research on the scheduling scheme during the peak period is a very important aspect and a very challenging subject in emergency logistics scheduling field. For solving this problem, we specially choose the time point of the peak period at 9 a.m. on Wednesday.

From Table 18, the time (in hours) and cost (ten thousand yuan as unit) consumptions of scheduling schemes are provided by algorithms. NSGA-II and MOEA/D can provide 6 schemes for decision makers to choose, while SPEA 2 can provide 8 schemes. Table 19 shows the average of time and cost; from the results, the schemes of MOEA/D and SPEA2 are dominated by NSGA-II's scheme. In transportation time, NSGA-II was $9 \%$ better than MOEA/D and $3 \%$ better than SPEA2; NSGA-II was $60 \%$ better than MOEA/D and 53\% better than SPEA2 in transportation cost. That means the better-quality solutions can be provided by NSGA-II.

From Table 20, the metrics spacing and PD show that the uniformity and diversity of solutions of NSGA-II are better than those in other algorithms, and the convergence of the algorithm is slightly worse than MOEA/D and SPEA2. Figure 8 shows the Pareto fronts of the three algorithms. 


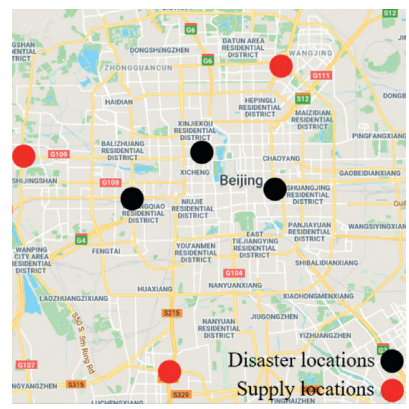

(a)

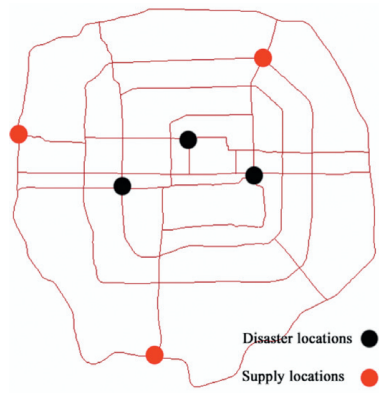

(b)

Figure 6: Supply locations and disaster locations. (a) The real map. (b) The vector map.

TABLE 13: The parameters of algorithm.

\begin{tabular}{lc}
\hline Parameter & Value \\
\hline Population & 100 \\
Iteration & 200 \\
Dimension & 3 \\
Crossover probability & 0.8 \\
Mutation probability & 0.2 \\
\hline
\end{tabular}

TABLE 14: The amount of relief supplies needed at the different disaster locations.

\begin{tabular}{lcc}
\hline Disaster location & Relief supplies & Amount $(\mathrm{kg})$ \\
\hline \multirow{3}{*}{1} & Food & 1540 \\
& Water & 2310 \\
& Medicine & 770 \\
\hline \multirow{3}{*}{2} & Food & 790 \\
& Water & 1184 \\
& Medicine & 395 \\
\hline \multirow{3}{*}{3} & Food & 2762 \\
& Water & 3642 \\
& Medicine & 1880 \\
\hline
\end{tabular}

TABLE 15: The fitness values (time and cost) of different algorithms at 4 a.m. on Tuesday.

\begin{tabular}{lccc}
\hline Scheme & NSGA-II & MOEA/D & SPEA2 \\
\hline 1 & $(0.6029,5.8804)$ & $(1.1307,14.5213)$ & $(0.9566,12.3205)$ \\
2 & $(0.6747,5.8768)$ & $(1.2020,14.4804)$ & $(0.9808,12.3109)$ \\
3 & $(3.2314,5.0696)$ & $(1.2728,14.1910)$ & $(0.9906,11.9888)$ \\
4 & $(3.3032,5.0660)$ & $(3.6864,13.2792)$ & $(1.0337,11.9488)$ \\
5 & - & $(3.7578,13.2382)$ & $(3.5851,11.5097)$ \\
6 & - & $(3.8286,12.9488)$ & - \\
\hline
\end{tabular}

TABle 16: The fitness values (time and cost) of different algorithms at 4 a.m. on Tuesday.

\begin{tabular}{lccc}
\hline & NSGA-II & MOEA/D & SPEA2 \\
\hline Average time & $1.9530 E+01$ & $2.4797 E+01$ & $1.5094 E+01$ \\
Average cost & $5.4732 E+04$ & $1.3776 E+05$ & $1.2016 E+05$ \\
\hline
\end{tabular}

From the rescue time of 4 a.m. on Tuesday and 9 a.m. on Wednesday, the contrast experiments show that the rescue time of 9 a.m. on Wednesday will spend much more
TABle 17: Comparisons of algorithms at 4 a.m. on Tuesday.

\begin{tabular}{lcccc}
\hline Algorithm & HV & Spacing & PD & Number \\
\hline NSGA-II & $2.40 E-01$ & $7.41 E-17$ & $1.03 E+03$ & 4 \\
MOEA/D & $4.41 E-01$ & $8.15 E-02$ & $1.06 E+03$ & 6 \\
SPEA2 & $6.62 E-01$ & $6.57 E-01$ & $8.95 E+02$ & 5 \\
\hline
\end{tabular}

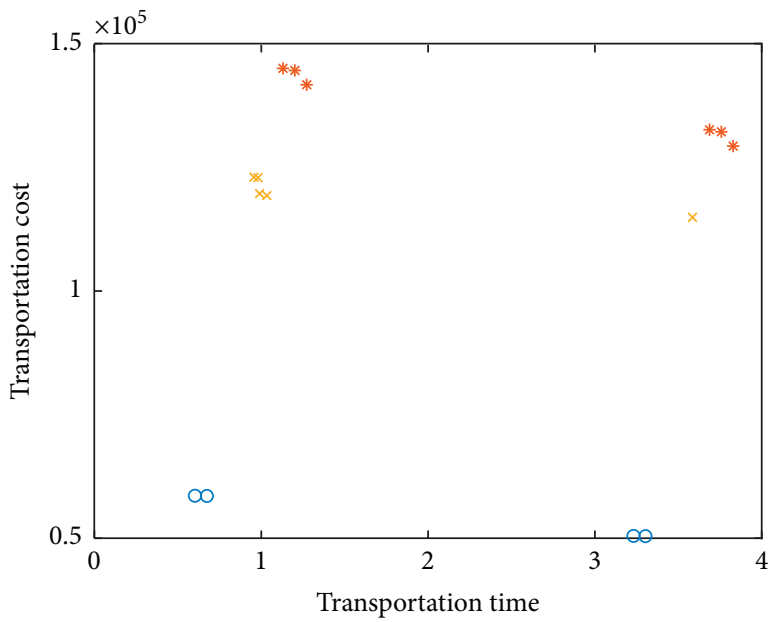

$$
\begin{aligned}
& \circ \text { NSGA-II } \\
& * \text { MOEA/D } \\
& \times \text { SPEA2 }
\end{aligned}
$$

FIgURe 7: The Pareto fronts of the three algorithms at 4 a.m. on Tuesday.

TABLE 18: The fitness values (time and cost) of different algorithms at 9 a.m. on Wednesday.

\begin{tabular}{lccc}
\hline Scheme & NSGA-II & MOEA/D & SPEA2 \\
\hline 1 & $(0.6709,6.0224)$ & $(1.2487,14.7557)$ & $(1.0516,12.3205)$ \\
2 & $(0.6787,5.8804)$ & $(1.2880,14.4203)$ & $(1.0594,12.1785)$ \\
3 & $(0.6902,5.8768)$ & $(1.3953,13.9389)$ & $(1.0709,12.1749)$ \\
4 & $(3.3464,5.2116)$ & $(1.4001,13.9198)$ & $(1.0873,11.8468)$ \\
5 & $(3.3542,5.0696)$ & $(3.9826,13.1654)$ & $(1.0988,11.8432)$ \\
6 & $(3.3657,5.0660)$ & $(3.9941,13.1618)$ & $(3.7350,11.3677)$ \\
7 & - & - & $(3.7464,11.3641)$ \\
8 & - & - & $(3.7628,11.0360)$ \\
\hline
\end{tabular}

transportation time and cost; that is, when the road is in crowded conditions, more time and cost are consumed. So, considering that the road conditions are necessary when 
TABle 19: The fitness values (time and cost) of different algorithms at 9 a.m. on Wednesday.

\begin{tabular}{lccc}
\hline & NSGA-II & MOEA/D & SPEA2 \\
\hline Average time & $2.0177 E+00$ & $2.2181 E+00$ & $2.0765 E+00$ \\
Average cost & $5.5211 E+04$ & $1.3894 E+05$ & $1.1766 E+05$ \\
\hline
\end{tabular}

TABLE 20: Comparisons of algorithms at 9 a.m. on Wednesday.

\begin{tabular}{lcccc}
\hline Algorithm & HV & Spacing & PD & Number \\
\hline NSGA-II & $3.67 E-01$ & $7.40 E-02$ & $9.09 E+02$ & 6 \\
MOEA/D & $7.17 E-01$ & $1.11 E-01$ & $8.74 E+02$ & 6 \\
SPEA2 & $5.82 E-01$ & $9.24 E-02$ & $7.65 E+02$ & 8 \\
\hline
\end{tabular}

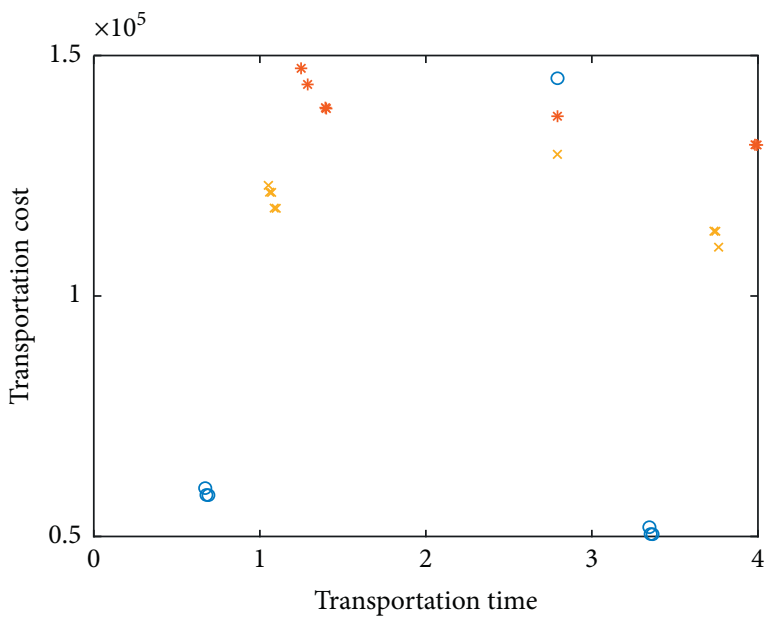

$$
\begin{aligned}
& \text { - NSGA-II } \\
& * \text { MOEA/D } \\
& \times \text { SPEA2 }
\end{aligned}
$$

Figure 8: Multiple Pareto fronts at 9 a.m. on Wednesday.

the emergency logistics scheduling model is established, the proposed model is more reasonable and it can provide more efficient scheduling under different traffic conditions.

4.2.3. The Rescue Time at 9 a.m. on Saturday. To test the impact of weekend traffic volume on emergency logistics scheduling model, we designed an experiment on the scheduling departure time in weekend. For a better comparison, 9 a.m. on Saturday is selected.

From Tables 21 and 22, the details of consumption and average consumption of transportation time and cost are given; from these results, the solutions of NSGA dominate the solutions given by the other two algorithms. NSGA-II is $36 \%$ better than MOEA/D and 37\% better than SPEA2 in transportation time; for transportation cost, the modified NSGA-II is $57 \%$ better than MOEA/D and $51 \%$ better than SPEA2. From Table 23, the numbers of plans solved by the three algorithms are roughly the same, but the plans of NSGA-II's metrics of spacing and PD are the best of the algorithms. Figure 9 shows the Pareto fronts; as can be seen
TABLe 21: Comparisons of algorithms at 9 a.m. on Wednesday.

\begin{tabular}{lccc}
\hline Scheme & NSGA-II & MOEA/D & SPEA2 \\
\hline 1 & $(0.8453,6.7140)$ & $(1.4758,15.2821)$ & $(1.3180,13.0121)$ \\
2 & $(0.8471,5.9532)$ & $(1.4819,15.1629)$ & $(1.3473,11.9196)$ \\
3 & $(0.8742,5.8768)$ & $(1.4837,12.2745)$ & $(1.6449,11.4405)$ \\
4 & $(1.1722,5.1424)$ & $(1.6995,11.0324)$ & $(1.6724,11.1088)$ \\
5 & $(1.1993,5.0660)$ & - & $(1.9101,11.0651)$ \\
\hline
\end{tabular}

TABle 22: The fitness values (time and cost) of different algorithms at 4 a.m. on Tuesday.

\begin{tabular}{lccc}
\hline & NSGA-II & MOEA/D & SPEA2 \\
\hline Average time & $9.8760 E-01$ & $1.5352 E+00$ & $1.5786 E+00$ \\
Average cost & $5.7505 E+04$ & $1.3438 E+05$ & $1.1709 E+05$ \\
\hline
\end{tabular}

TABLE 23: Comparisons of algorithms at 9 a.m. on Wednesday.

\begin{tabular}{lcccc}
\hline Algorithm & HV & Spacing & PD & Number \\
\hline NSGA-II & $7.46 E-01$ & $1.54 E-01$ & $8.17 E+02$ & 5 \\
MOEA/D & $8.93 E-01$ & $5.79 E-01$ & $6.78 E+02$ & 4 \\
SPEA2 & $9.22 E-01$ & $1.97 E-01$ & $7.47 E+02$ & 5 \\
\hline
\end{tabular}

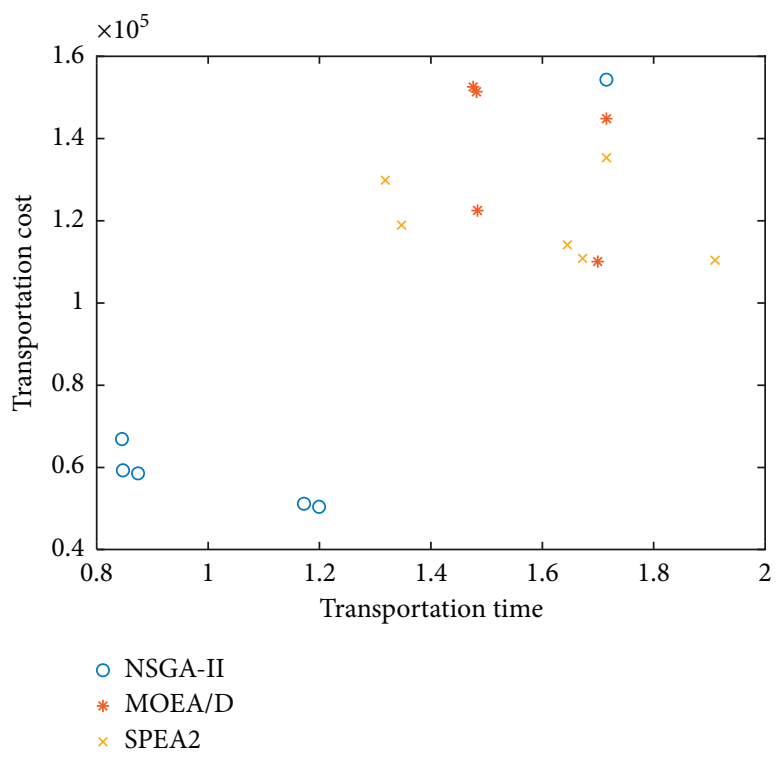

Figure 9: Multiple Pareto fronts at 9 a.m. on Saturday.

from the figure, the results of NSGA-II dominate the other two algorithms' results.

In this section, due to the uncertain time of emergency logistics scheduling, three different departure times are selected for simulation experiments. The roadway congestion indexes are uncertain given by cloud model in these departure times, and the scheduling of different departure times is also different.

In order to display the dispatching scheduling route intuitively, we chose 9 a.m. on Wednesday as the departure time of rescue. We solved the model with three algorithms, NSGA-II, MOEA/D, and SPEA2, and selected three algorithms to provide detailed scheduling route at the inflection point of Pareto frontier. Figures $10(\mathrm{a})-10$ (c) represent the 


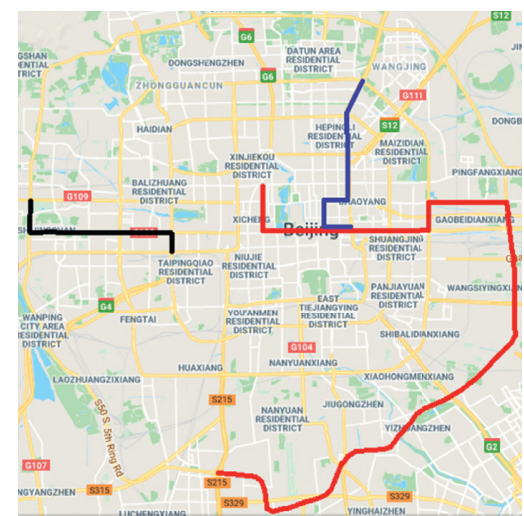

(a)

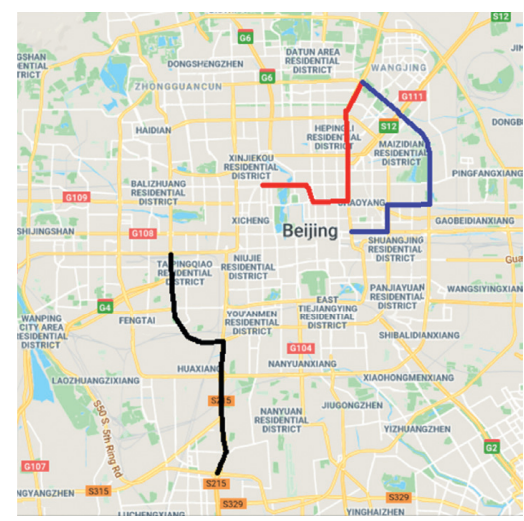

(b)

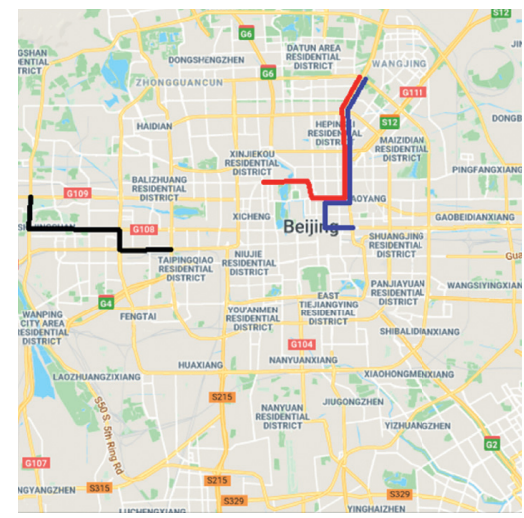

(c)

Figure 10: Scheduling of different algorithms at 9 a.m. on Wednesday.

scheduling route of NSGA-II, MOEA/D, and SPEA2, respectively.

\section{Conclusions and Future Work}

This paper proposed a biobjective emergency logistics scheduling model based on uncertain traffic conditions. First, the highly uncertain traffic conditions are converted into quantitative traffic volume by using the one-dimensional cloud model; experiments show that the cloud model can provide accurate prediction and adapt to the fuzziness and randomness of road traffic. One-dimensional cloud model converts traffic volume into the roadway congestion index, and, on this basis, an emergency logistics scheduling model with two objectives of transportation time and cost is constructed. Meanwhile, the multiobjective NSGA-II algorithm is improved to solve the proposed model. The experimental results show that it can provide a series of effective scheduling schemes, by taking into account different departure times and traffic conditions. At 4 a.m. on Tuesday, NSGA-II dominates MOEA/D, but NSGA-II is $29 \%$ worse than SPEA2 in the first objective; NSGA-II is $54 \%$ better than SPEA2 in the second objective; NSGA-II was $9 \%$ better than MOEA/D and 3\% better than SPEA2; NSGA-II was $60 \%$ better than MOEA/D and $53 \%$ better than SPEA2 in transportation cost at 9 a.m. on Wednesday; NSGA-II was $36 \%$ better than MOEA/D and 37\% better than SPEA2 in transportation time; for transportation cost, and the modified NSGA-II wass $57 \%$ better than MOEA/D and $51 \%$ better than SPEA2 at 9 a.m. on Saturday. The proposed model is more reasonable because it takes into account uncertain traffic conditions, which can improve the rescue efficiency and shorten the rescue time.

In addition, according to the problems existing in emergency logistics scheduling, how to construct road safety and resource distribution satisfaction in our proposed model will be our next work. Meanwhile, for the accuracy of prediction, how to build a two-dimensional cloud model based on traffic data will also be our next work.

\section{Data Availability}

As the project has not been completed, the experimental code and data cannot be disclosed for the time being. If researchers need the project code, please contact the corresponding author.

\section{Conflicts of Interest}

The authors declare that there are no conflicts of interest.

\section{Acknowledgments}

This work was supported by the National Natural Science Foundation of China under Grant no. 61806138 and Key R\&D program of Shanxi Province (High Technology) under Grant no. 201903D121119.

\section{References}

[1] A. Ben-Tal, D. den Hertog, A. De Waegenaere, B. Melenberg, and G. Rennen, "Robust solutions of optimization problems affected by uncertain probabilities," Management Science, vol. 59, no. 2, pp. 341-357, 2013.

[2] H. Chi, J. Li, X. Shao, and M. Gao, "Timeliness evaluation of emergency resource scheduling," European Journal of Operational Research, vol. 258, no. 3, pp. 1022-1032, 2017.

[3] J. R. Batmetan, A. J. Santoso, and P. Pranowo, "A multipleobjective ant colony algorithm for optimizing disaster relief logistics," Advanced Science Letters, vol. 23, no. 3, pp. 23442347, 2017.

[4] X. Gan and J. Liu, "A multi-objective evolutionary algorithm for emergency logistics scheduling in large-scale disaster 
relief," in Proceedings of the 2017 IEEE Congress on Evolutionary Computation, pp. 51-58, San Sebastian, Spain, June 2017.

[5] Y. Zhou, G. Zhou, and R. Zhao, "Solving large-scale 0-1 knapsack problem by the social-spider optimisation algorithm," International Journal of Computing Science and Mathematics, vol. 9, no. 5, pp. 433-441, 2018.

[6] A. Ben-Tal, B. D. Chung, S. R. Mandala, and T. Yao, "Robust optimization for emergency logistics planning: risk mitigation in humanitarian relief supply chains," Transportation Research Part B: Methodological, vol. 45, no. 8, pp. 1177-1189, 2011.

[7] Z. Cui, F. Xue, X. Cai, Y. Cao, G.-G. Wang, and J. Chen, "Detection of malicious code variants based on deep learning," IEEE Transactions on Industrial Informatics, vol. 14, no. 7, pp. 3187-3196, 2018.

[8] S. Tapaswini and S. Chakraverty, "Numerical solution of fuzzy differential equations using orthogonal polynomials," International Journal of Computing Science and Mathematics, vol. 10, no. 1, pp. 32-45, 2019.

[9] X. Cai, P. Wang, L. Du, Z. Cui, W. Zhang, and J. Chen, "Multiobjective three-dimensional DV-hop localization algorithm with NSGA-II," IEEE Sensors Journal, vol. 19, no. 21, pp. 10003-10015, 2019.

[10] X. Cai, X. Z. Gao, and Y. Xue, "Improved bat algorithm with optimal forage strategy and random disturbance strategy," International Journal of Bio-Inspired Computation, vol. 8, no. 4, pp. 205-214, 2016.

[11] X. Cai, Y. Niu, S. Geng et al., "An under-sampled software defect prediction method based on hybrid multi-objective cuckoo search," Concurrency and Computation: Practice and Experience, 2019.

[12] Z. Cui, M. Zhang, H. Wang, X. Cai, and W. Zhang, "A hybrid many-objective cuckoo search algorithm," Soft Computing, vol. 23, no. 21, pp. 10681-10697, 2019.

[13] J. Zhang, F. Xue, X. Cai et al., "Privacy protection based on many-objective optimization algorithm," Concurrency and Computation Practice and Experience, vol. 31, no. 20, Article ID e5342, 2019.

[14] X. Cai, S. Geng, D. Wu, L. Wang, and Q. Wu, "A unified heuristic bat algorithm to optimize the LEACH protocol," Concurrency and Computation: Practice and Experience, 2019.

[15] X. Cai, J. Zhang, H. Liang, L. Wang, and Q. Wu, "An ensemble bat algorithm for large-scale optimization," International Journal of Machine Learning and Cybernetics, vol. 10, no. 11, pp. 3099-3113, 2019.

[16] Z. Cui, L. Du, P. Wang, X. Cai, and W. Zhang, "Malicious code detection based on CNNs and multi-objective algorithm," Journal of Parallel and Distributed Computing, vol. 129, pp. 50-58, 2019.

[17] X. Cai, M. Zhang, H. Wang, M. Xu, J. Chen, and W. Zhang, "Analyses of inverted generational distance for many-objective optimisation algorithms," International Journal of BioInspired Computation, vol. 14, no. 1, pp. 62-68, 2019.

[18] Z. Cui, Y. Cao, X. Cai, J. Cai, and J. Chen, "Optimal LEACH protocol with modified bat algorithm for big data sensing systems in Internet of Things," Journal of Parallel and Distributed Computing, vol. 132, pp. 217-229, 2019.

[19] M. W. P. Savelsbergh, "Local search in routing problems with time windows," Annals of Operations Research, vol. 4, no. 1, pp. 285-305, 1985.

[20] J. Li, Y. X. Li, S. S. Tian, and J. Zou, "Dynamic cuckoo search algorithm based on Taguchi opposition-based search,"
International Journal of Bio-Inspired Computation, vol. 13, no. 1, pp. 59-69, 2019.

[21] Q. Wang, "Improve TSP problem allowing multiple vehicles distribution," in Proceedings of the 2016 International Conference on Logistics Informatics and Service Sciences (LISS), pp. 1-3, Sydney, NSW, Australia, February 2017.

[22] M. Jingyan and Z. Kehong, "Research on TSP solution based on genetic algorithm of Logistic equation," in Proceedings of the 2012 2nd International Conference on Computer Science and Network Technology, pp. 738-742, Changchun, China, December 2012.

[23] Z. Gong and Z. Li, "Intelligent control Technology of quadrotor UAV based on one-dimensional cloud model," Command Control and Simulation, vol. 40, no. 5, pp. 106-111, 2018.

[24] K. Deb, A. Pratap, S. Agarwal, and T. Meyarivan, "A fast and elitist multiobjective genetic algorithm: NSGA-II," IEEE Transactions on Evolutionary Computation, vol. 6, no. 2, pp. 182-197, 2002.

[25] Q. Zhang and H. Li, "MOEA/D: a multiobjective evolutionary algorithm based on decomposition," IEEE Transactions on Evolutionary Computation, vol. 11, no. 6, pp. 712-731, 2007.

[26] M. Kim, T. Hiroyasu, M. Miki, and S. Watanabe, "SPEA2+: improving the performance of the strength pareto evolutionary algorithm 2," in Parallel Problem Solving from Nature-PPSN VIII, LNCS, vol. 3242, pp. 742-751, Springer, Berlin, Germany, 2004.

[27] G. Chai, J. Cao, W. Huang, and J. Guo, "Optimized traffic emergency resource scheduling using time varying rescue route travel time," Neurocomputing, vol. 275, pp. 1567-1575, 2018.

[28] J. Li, J.-H. Zhang, and D.-L. Zhu, "Multi-resource emergency scheduling model and algorithm in disaster chain," Systems Engineering-Theory \& Practice, vol. 31, no. 3, pp. 488-495, 2011.

[29] Q. Wang, C. Zhu, Y. Li, and Z. Zhang, "Research on robust optimization of emergency logistics network considering the time dependence characteristic," in Proceedings of the International Conference on AEECE, vol. 69, no. 1, Chengdu, China, June 2017.

[30] R. Wang, M. Qi, and A. Han, "Comparative study of emergency logistics path planning," Journal of Donghua University, vol. 35, no. 3, pp. 274-278, 2018.

[31] H. Abbasnejad and A. Jafarian, "A new method based on artificial neural networks for solving general nonlinear systems," International Journal of Computing Science and Mathematics, vol. 9, no. 3, pp. 207-218, 2018.

[32] D. Gonidakis, "Symbiotic organisms search algorithm for different economic load dispatch problems," International Journal of Bio-Inspired Computation, vol. 12, no. 3, pp. 139151, 2018.

[33] G. Duan, L. Chen, Y. Z. Li, and R. C. He, "Optimisation on empty trains distribution with time window in heavy haul railway," International Journal of Computing Science and Mathematics, vol. 9, no. 3, pp. 273-286, 2018.

[34] P. K. Pandey, "An efficient numerical method for the solution of third order boundary value problem in ordinary differential equations," International Journal of Computing Science and Mathematics, vol. 9, no. 2, pp. 171-180, 2018.

[35] T. Yigit, O. Unsal, and O. Deperlioglu, "Using the metaheuristic methods for real-time optimisation of dynamic school bus routing problem and an application," International Journal of Bio-Inspired Computation, vol. 11, no. 2, pp. 123133, 2018. 
[36] M. Sajid, Z. Raza, and M. Shahid, "Hybrid bio-inspired scheduling algorithms for batch of tasks on heterogeneous computing system," International Journal of Bio-Inspired Computation, vol. 11, no. 3, pp. 135-148, 2018.

[37] W. Shu, W. Qian, and J. Yang, "An efficient approximation algorithm for the extension facility location problem on torus internetwork topology," International Journal of Computing Science and Mathematics, vol. 9, no. 2, pp. 189-196, 2018.

[38] H. Wu, Y. Zhou, and Q. Luo, "Hybrid symbiotic organisms search algorithm for solving 0-1 knapsack problem," International Journal of Bio-Inspired Computation, vol. 12, no. 1, pp. 23-53, 2018.

[39] Z. Cui, Y. Chang, J. Zhang, X. Cai, and W. Zhang, "Improved NSGA-III with selection-and-elimination operator," Swarm and Evolutionary Computation, vol. 49, pp. 23-33, 2019.

[40] Z. Cui, J. Zhang, Y. Wang et al., "A pigeon-inspired optimization algorithm for many-objective optimization problems," Science China Information Sciences, vol. 62, no. 7, 2019.

[41] X. Ren, J. Zhu, and J. Huang, "Multi-period dynamic model for emergency resource dispatching problem in uncertain traffic network," Systems Engineering Procedia, vol. 5, pp. 37-42, 2012.

[42] Y. Deng, W. Zhu, and F. Wang, "Research on multi-objective robust vehicle routing problem in emergency logistics," Computer Engineering and Applications, vol. 55, no. 1, pp. 248-255, 2019.

[43] Y. Zhou, J. Liu, Y. Zhang, and X. Gan, "A multi-objective evolutionary algorithm for multi-period dynamic emergency resource scheduling problems," Transportation Research Part E: Logistics and Transportation Review, vol. 99, pp. 77-95, 2017.

[44] Q. Chang, "Vehicle scheduling model of emergency logistics distribution based on internet of things," International Journal of Applied Decision Sciences, vol. 11, no. 1, pp. 36-54, 2018.

[45] Z. Ding, X. Li, Y. Cao, R. Yao, Y. Chi, and L. Guo, “An effective emergency logistics scheduling model based on multiobjective optimization algorithms," in Proceedings of the 4th ACM SIGSPATIAL International Workshop on Safety and Resilience-Safety and Resilience'18, Seattle, WA, United States, November 2018.

[46] D. Li, H. Meng, and X. Shi, "Membership clouds and membership cloud generator," Journal of Computer Research and Development, vol. 32, no. 6, pp. 15-20, 1995.

[47] D. Li and C. Liu, "Study on the universality of the normal cloud model," Engineering Science, vol. 6, no. 8, pp. 28-34, 2004.

[48] S.-W. Yu, K. Cao, and M. Zhao, "Traffic information forecast algorithm based on the one-dimension cloud model," Journal of Shandong University, vol. 37, no. 2, pp. 121-126, 2007.

[49] M. Zhao, K. Cao, and S. Ho, "Real-time traffic prediction using AOSVR and cloud model," in Proceedings of the 2007 IEEE Intelligent Transportation Systems Conference, pp. 485489, Bellevue, WA, USA, September 2007.

[50] Q. Liu and J. Xu, "Research on prediction of the short-term traffic flow based on cloud model," Computer Engineering and Design, vol. 33, no. 5, pp. 1953-1957, 2012. 